\title{
Measurement of parton shower observables with OPAL
}

\author{
N. Fischer ${ }^{1}$, S. Gieseke ${ }^{2}$, S. Kluth ${ }^{3, a}$, S. Plätzer ${ }^{4}$, P. Skands ${ }^{1}$, and OPAL collaboration \\ ${ }^{1}$ School of Physics and Astronomy, Monash University, Melbourne, Australia \\ ${ }^{2}$ Institute for Theoretical Physics, Karlsruhe Institute of Technology, Karlsruhe, Germany \\ ${ }^{3}$ Max-Planck-Institute for Physics, Munich, Germany \\ ${ }^{4}$ Institute for Particle Physics Phenomenology, Durham University, Durham, United Kingdom
}

\begin{abstract}
A study of QCD coherence is presented based on a sample of about 397,000 e+e- hadronic annihilation events collected at $\sqrt{s}=91 \mathrm{GeV}$ with the OPAL detector at LEP. The study is based on four recently proposed observables that are sensitive to coherence effects in the perturbative regime. The measurement of these observables is presented, along with a comparison with the predictions of different parton shower models. The models include both conventional parton shower models and dipole antenna models. Different ordering variables are used to investigate their influence on the predictions.
\end{abstract}

\section{Introduction}

In the evolution of a hard hadronic interaction to a final state observable in experiment the concept of the parton shower plays an important role. The parton shower connects the few energetic partons coming out of a hard scattering with the many soft partons thought to enter the hadronisation phase where observable hadrons are created. The main ingredient of the parton shower is that a hard parton transforms into a jet by repeatedly radiating gluons, which themselves can radiate, or create a quark-antiquark-pair, thus creating an avalanche or shower of partons. This process is regulated by colour coherence, i.e. the destructive interference of multiple colour-connected soft gluon emissions. The iterative nature of this process together with a simple probabilistic interpretation of each vertex or parton branching in the leading logarithmic approximation is the basis for many successful implementations in event based Monte Carlo simulation programs. A recent review about this subject is e.g. [1].

For many areas of particle physics the availability of universal numerical predictions for many different observables via Monte Carlo event generators is essential. For example at the LHC predictions for jet based observables like the distribution of the transverse momentum values of the most energetic jets in each event is usually calculated with an event generator program. Such a program contains calculations for the hard scattering of partons from the two colliding protons, a parton shower algorithm to model the evolution the energetic partons to jets of many soft partons, and a hadronisation model to describe the transition of partons to hadrons. The availability of next-to-leading order (NLO) calculations for complex final states in the event generators with consistent matching to the parton shower models has improved the accuracy of such predictions significantly. In turn, the theoretical accuracy

ae-mail: skluth@mpp.mpg.de 
of the parton shower models has become a topic of interest for theorists in order to provide the best possible predictions.

It is difficult to construct decisive tests with the aim to distinguish between different parton shower model implementations in terms of their ability to describe data correctly. In [2] new observables based on $\mathrm{e}^{+} \mathrm{e}^{-}$annihilation into hadrons are proposed and studied to investigate differences between parton shower models. These new observables were shown to have sensitivity to explore differences between parton shower models and therefore a measurement with data from the OPAL experiment at LEP was performed [3].

\section{Theory background}

\subsection{4-jet topology observables}

The new observables are based on selecting 4-jet final states from $\mathrm{e}^{+} \mathrm{e}^{-}$annihilation to hadronic final states at a centre-of-mass energy of $\sqrt{s}=91 \mathrm{GeV}$ corresponding to the $\mathrm{Z}^{0}$ peak. The jets are reconstructed using the Durham iterative jet clustering algorithm [4]. The phase-space distance $y_{i j}$ between any two particles $i, j$ with energies $E_{i, j}$ and angle $\theta_{i j}$ between them is defined by $y_{i j}=2 \min \left(E_{i}, E_{j}\right)^{2}\left(1-\cos \theta_{i j}\right) / s$. The pair with the smallest value of $y_{i j}$ is replaced by a pseodoparticle obtained by adding the 4-vectors; this procedure is repeated until two pseodo-particles or jets remain. The value of $y_{i j}$ found when going from a 4-jet to a 3-jet configuration is required to be $y_{43}>0.0045$. In the 4 -jet configuration the jets are ordered with decreasing energy values counting from 1 to 4 .

The four observables used in the analysis can now be explained. For the first three observables the angles between the jets are subjected to the requirements $\theta_{12}>2 \pi / 3, \theta_{13}>2 \pi / 3$ and $\theta_{23}<\pi / 6$.

$\boldsymbol{\theta}_{\mathbf{1 4}}$ : The observable $\theta_{14}$ is defined as the angle between jets 1 and 4 , as shown in figure 1 a) [5].

$\boldsymbol{\theta}^{*}$ : With the additional requirement $\theta_{24}<\pi / 2$ the observable $\theta^{*}=\theta_{24}-\theta_{23}$ is defined, see also figure $1 \mathrm{~b})$ [6].

$C_{\mathbf{2}}^{(\mathbf{1 / 5 )}}$ : The so-called 2-point energy correlation double ratio [7].

$\rho$ : For the observable $\rho$ instead of restricting the angles between the jets the requirement $y_{43}>0.5 \cdot y_{32}$ for the values of $y_{i j}$ for going from a 4-jet to a 3-jet and for going from a 3-jet to a 2-jet configuration is made. In the final 2-jet configuration the invariant masses in both jets are calculated and denoted $M_{H}$ for the larger and $M_{L}$ for the smaller value. The ratio of these hemisphere masses $\rho=\left(M_{L} / M_{H}\right)^{2}$ defines the fourth observable [5]. Configurations corresponding to a small or large value of $\rho$ are shown in figures $1 \mathrm{c}$ ) and $\mathrm{d}$ ).

All observables are constructed to be sensitive to coherence effects and other properties of the parton shower models.

\subsection{Parton shower models}

We give a brief overview over the implementations of parton shower models in modern Monte Carlo generator programs, which will be used to produce predictions for comparison with measurements of the new observables explained above. In total six different parton shower implementations are studied, where three are variants of the Herwig++ program [8] and three are variants of the Pythia8 program [9] using the VINCIA plugin [10].

From the Herwig++ program we consider three different implementations of the parton shower: 
a)

2

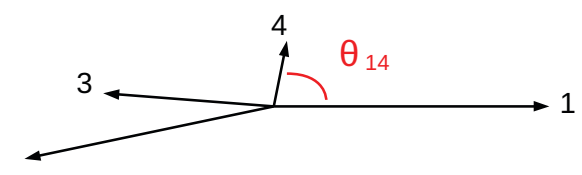

c)

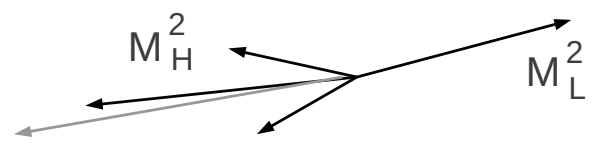

b)

2
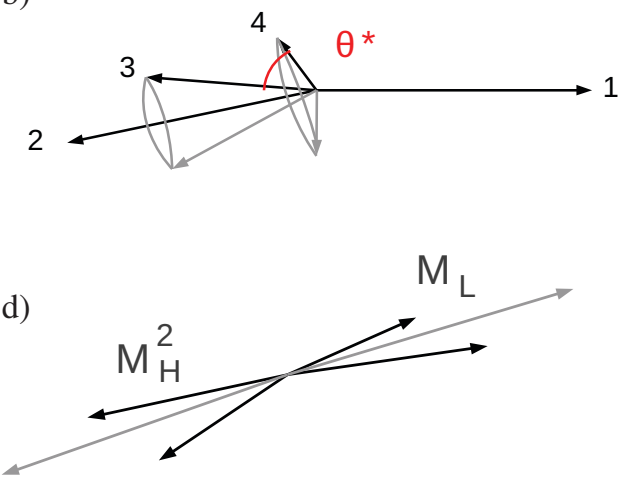

Figure 1. Figure a) shows the definition of $\theta_{14}$ as the angle between the most and least energetic jets in the 4-jet event with kinematic constraints. Figure b) displays the observable $\theta^{*}$ as the difference between angles $\theta_{23}$ and $\theta_{24}$. Figure c) represents a configuration where three lower energy jets recoil against one energetic jet leading to small values of $\rho$ while figure d) gives a configuration with two pairs of close jets recoiling against each other leading to $\rho \approx 1$.

$\tilde{q}^{2}$ ordering: The evolution of the parton shower is based on a DGLAP kernel with ordering variable $\tilde{q}^{2}$ proportional to the product of energy and angle.

$p_{\perp, \text { dip }}^{2}$ ordering: The partons shower model is based on Catani-Seymour (CS) dipoles and uses as ordering variable the relative transverse momentum of the splitting pair $p_{\perp, d i p}^{2}$.

$\boldsymbol{q}_{\text {dip }}^{2}$ ordering: The same parton shower model based on CS dipoles is used but with the invariant mass or virtuality of the splitting pair $q_{d i p}^{2}$ as ordering variable. This shower model is expected to take colour coherence effects less well into account.

The VINCIA plugin for Pythia8 implements a parton shower based on antenna functions with local recoils within the antennae while the regular Pythia8 parton shower model is based on DGLAP splitting kernels. From the Pythia8 program and the VINCIA plugin we have the following implementations of the parton shower:

VINCIA $p_{\perp, \text { ant }}^{2}$ ordering: The shower model uses $p_{\perp, \text { ant }}^{2}$ without matrix element corrections. The shower model takes colour coherence into account through the antenna function formalism. This option is the preferred choice for the VINCIA shower [11].

VINCIA $\boldsymbol{m}_{\text {ant }}^{2}$ ordering: In this variation of the VINCIA parton shower as above the ordering variable is defined as the antenna mass $m_{\text {ant }}^{2}$.

Pythia8 $p_{\perp, \text { evol }}^{2}$ ordering: The regular Pythia8 shower model based on DGLAP splitting kernels is ordered in relative transverse momentum $p_{\perp, \text { evol }}^{2}$ of the parton pair, applies a LO matrix element correction and uses vetos on emission angles to be colour coherent.

The six different Monte Carlo models are tuned to the same set of observables measured in $\mathrm{e}^{+} \mathrm{e}^{-}$ annihilation at LEP [2]. In this way differences between the models due to different quality of description of the data are removed. 


\section{Data analysis}

We analyse data taken with the OPAL experiment at the LEP $\mathrm{e}^{+} \mathrm{e}^{-}$collider at centre-of-mass $(\mathrm{cms})$ energies near the $Z^{0}$ peak $\sqrt{s} \approx 91 \mathrm{GeV}$. The momenta of charged particle tracks are measured with the so-called jet chamber while the energies of all particles are measured from clusters of hit blocks of the lead glass electro-magnetic calorimeter; for details please see [3] and references therein. The tracking and calorimeter detectors provided almost complete coverage of the solid angle and in addition the event selection ensures well contained events. After the standard OPAL selection of hadronic events those with special 4-jet topology are selected as explained above in section 2.1.

In order to perform corrections for acceptance of the event selection and for resolution effects samples of simulated events are used. The simulations are based on Monte Carlo generators for $\mathrm{e}^{+} \mathrm{e}^{-}$ annihilation into hadrons as used by the OPAL collaboration in its active period [12,13] combined with a detailed simulation of the OPAL experiment [14]. These generators used parameter sets determined by the OPAL collaboration from tuning to OPAL data. The simulated events were processed with the same reconstruction and analysis software as the data. In simulated events we refer to results derived from the observed data as "detector-level" and to results derived from the output of the generators after hadronisation and decays of short-lived particles as "hadron-level"1. The experimental correction uses iterative bayesian unfolding [15] based on a response matrix derived from comparing event-by-event results at the detector- and hadron-level derived from the simulations.

It is observed that the distributions of the four observables at the detector level are well reproduced by the simulated data. As systematic variations of the analysis selection cuts are varied, the response matrix is calculated based on a different generator and the unfolding method is changed. The systematic variations are dominated by the effect of calculating the response matrix using a different generator and are larger than the statistical errors.

As a cross check the predictions of the simulations samples based on old Monte Carlo generators used for the experimental corrections are compared with the corrected data and good consistency within the combined statistical and experimental uncertainties is observed.

\section{Comparison of parton shower models with data}

The new parton shower models as implemented in Herwig++, Pythia8/VINCIA or Pythia8 are run with large statistics ( $5 \cdot 10^{6}$ events) and are compared to the corrected data at the hadron-level.

Figure 2 shows in both panels the same corrected distribution of $\theta_{14}$ measured by OPAL with statistical and experimental uncertainties. In figure 2 a) the predictions of the Herwig++ variants and on figure $2 \mathrm{~b}$ ) the predictions of the Pythia8/VINCIA variants are shown. In all cases the description of the data by the predictions is adequate with the exception of a narrow region $\theta_{14} \approx 0.7 \pi$ where Herwig $++p_{\perp, \text { dip }}^{2}$ deviates by about three standard deviations.

Figure 3 shows on both panels the asymmetry $N_{\text {left }} / N_{\text {right }}$ of the distribution of $\rho$, which is defined by $N_{\text {left }} / N_{\text {right }}=\sum_{x_{i}<x_{0}} n_{i} / \sum_{x_{i}>x_{0}} n_{i}$ with bin centers $x_{i}$, bin contents $n_{i}$ and dividing point $x_{0}$. The asymmetry is shown for two dividing points $\rho_{0}$. We observe comparatively large differences between the Herwig++ parton shower model predictions. We also find that the Herwig $++\tilde{q}^{2}$ model provides among the Herwig++ variants the best description of the data while the Herwig $++q_{d i p}^{2}$ model disagrees by up to four standard deviations with the data. The Pythia8/VINCIA model predictions lie closer together and also provide a reasonable description of the data.

In [3] comparisons to distributions and asymmetries of all four observables can be found together with tables of the data, their uncertainties and correlations.

\footnotetext{
${ }^{1}$ Hadron-level is often also referred to as particle-level.
} 
a) Angle between 1 st and 4 th jet, $\theta_{14}$, HerwiG++

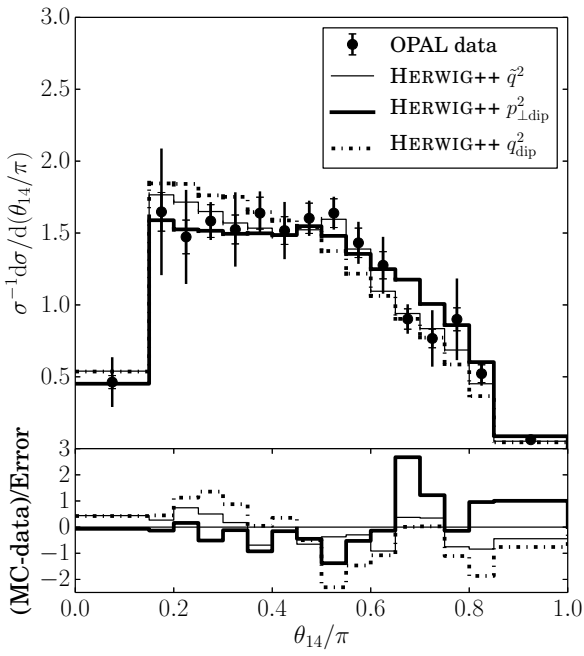

b) Angle between 1 st and 4 th jet, $\theta_{14}$, Vincia, Pythia 8

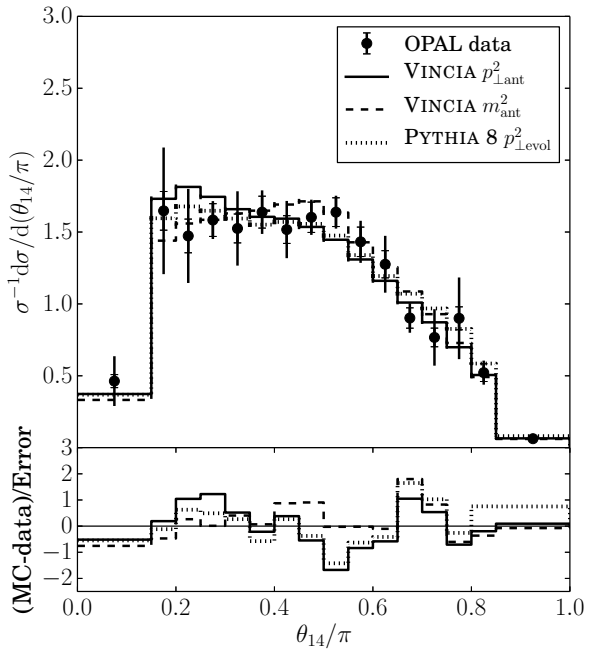

Figure 2. Figure a) shows as points with error bars the corrected distribution of $\theta_{14}$ with total errors. The statistical errors are indicated by the small horizontal bars. The lines represent the three variants of Herwig++ models as indicated. Figure b) shows the same data with the lines representing the three Pythia8 model predictions as indicated.

\section{Conclusions and outlook}

In general we find that all models give a fair description of the data measured by OPAL. We also observe discrepancies of up to four standard deviations for some predictions in some restricted regions of the asymmetries of the observables. These observations confirm that the new observables can have more sensitivity w.r.t. parton shower model differences compared with traditional observables. The Herwig $++q_{d i p}^{2}$ model gives the least satisfactory description of the data; this is expected since this model on purpose takes coherence effects less well into account.

In this study the Herwig++ and Pythia8 variants use the cluster or string model for hadronisation, respectively, which limits the strength of our conclusions. It would be valuable to repeat the comparison with the data with all parton shower models coupled to the same hadronisation model.

\section{References}

[1] S. Höche, Introduction to parton-shower event generators, in Theoretical Advanced Study Institute in Elementary Particle Physics: Journeys Through the Precision Frontier: Amplitudes for Colliders (TASI 2014) Boulder, Colorado, June 2-27, 2014 (2014), 1411.4085

[2] N. Fischer, S. Gieseke, S. Plätzer, P. Skands, Eur. Phys. J. C74, 2831 (2014), 1402 . 3186

[3] N. Fischer, S. Gieseke, S. Kluth, S. Plätzer, P. Skands (OPAL), Eur. Phys. J. C75, 571 (2015), 1505.01636

[4] S. Catani et al., Phys. Lett. B, 432 (1991)

[5] J. Alcaraz Maestre et al. (SM MC Working Group, SM and NLO MULTILEG Working Group), The SM and NLO Multileg and SM MC Working Groups: Summary Report, in Proceedings, 7th Les Houches Workshop on Physics at TeV Colliders (2012), pp. 1-220, 1203.6803 
c) Asymmetry for $\rho$, HeRwiG++

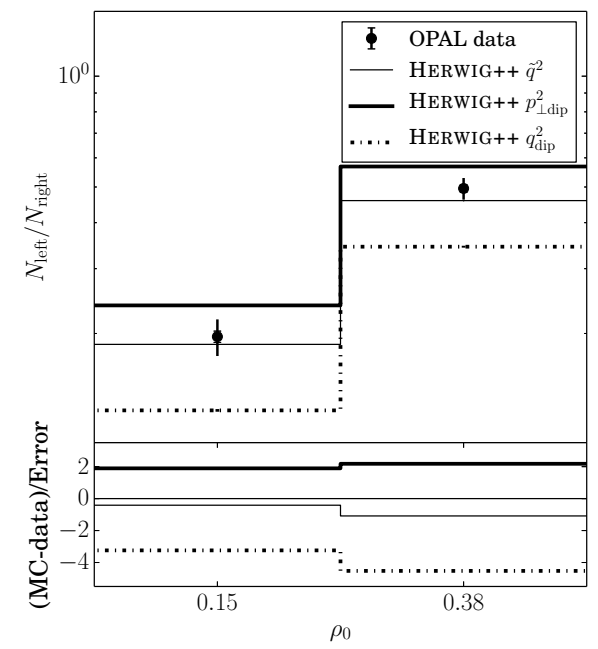

d) Asymmetry for $\rho$, Vincia, Pythia 8

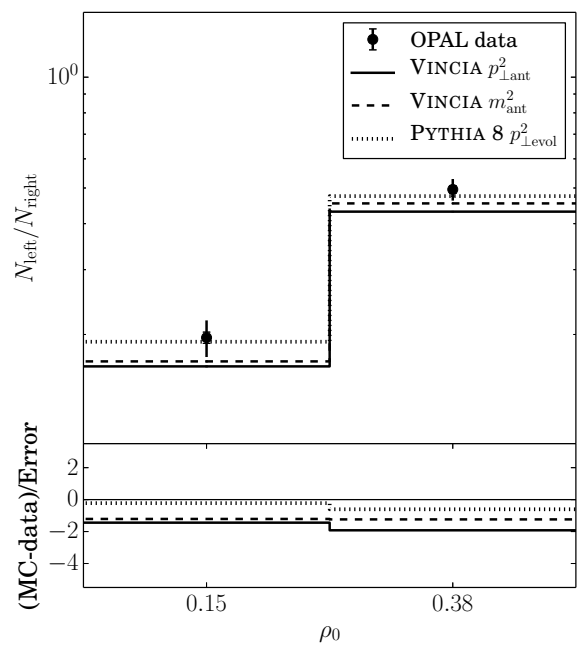

Figure 3. Figure c) shows as points with error bars the corrected asymmetry distribution (see text) of $\rho$ with total uncertainties. The small horizontal bars give the statistical uncertainties. The lines display the predictions by the three Herwig++ models as indicated. Figure d) shows the same data compared with the three Pythia8 models as lines as indicated on the figure.

[6] S. Platzer, S. Gieseke, J. High Energy Phys. 01, 024 (2011), 0909. 5593

[7] A.J. Larkoski, G.P. Salam, J. Thaler, J. High Energy Phys. 06, 108 (2013), 1305.0007

[8] S. Gieseke, P. Stephens, B. Webber, J. High Energy Phys. 12, 045 (2003), hep-ph/0310083

[9] T. Sjöstrand, S. Mrenna, P. Skands, Comput. Phys. Commun. 178, 852 (2008), 0710 . 3820

[10] W.T. Giele, D.A. Kosower, P.Z. Skands, Phys. Rev. D, 014026 (2008), 0707 . 3652

[11] L. Hartgring, E. Laenen, P. Skands, J. High Energy Phys. 10, 127 (2013), 1303.4974

[12] T. Sjöstrand, Comput. Phys. Commun. 82, 74 (1994)

[13] G. Marchesini et al., Comput. Phys. Commun. 67, 465 (1992)

[14] J. Allison et al., Nucl. Instrum. Methods A, 47 (1992)

[15] G. D’Agostini, Nucl. Instrum. Methods A, 487 (1995) 